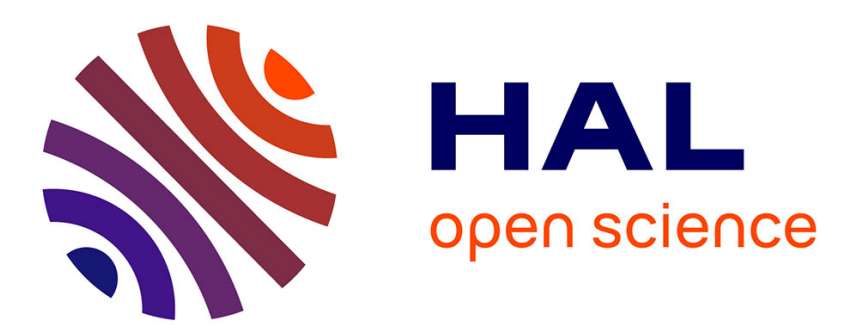

\title{
Anisotropic phase-mixing in homogeneous turbulence in a rapidly rotating or in a strongly stratified fluid: An analytical study
}

\author{
Abdelaziz Salhi, Claude Cambon
}

\section{- To cite this version:}

Abdelaziz Salhi, Claude Cambon. Anisotropic phase-mixing in homogeneous turbulence in a rapidly rotating or in a strongly stratified fluid: An analytical study. Physics of Fluids, 2007, 19, pp.055102. 10.1063/1.2728934 . hal-00272172

\section{HAL Id: hal-00272172}

\section{https://hal.science/hal-00272172}

Submitted on 14 Jun 2012

HAL is a multi-disciplinary open access archive for the deposit and dissemination of scientific research documents, whether they are published or not. The documents may come from teaching and research institutions in France or abroad, or from public or private research centers.
L'archive ouverte pluridisciplinaire HAL, est destinée au dépôt et à la diffusion de documents scientifiques de niveau recherche, publiés ou non, émanant des établissements d'enseignement et de recherche français ou étrangers, des laboratoires publics ou privés. 


\title{
Anisotropic phase-mixing in homogeneous turbulence in a rapidly rotating or in a strongly stratified fluid: An analytical study
}

\author{
A. Salhi \\ Département de Physique, Faculté des Sciences de Tunis, Université de Tunis El-Manar, 1060, Tunis, \\ Tunisia \\ C. Cambon \\ Laboratoire de Mécanique des Fluides et d'Acoustique, École Centrale de Lyon/UCB Lyon/INSA/CNRS, \\ 69134 Ecully Cedex, France
}

(Received 11 August 2006; accepted 1 March 2007; published online 1 May 2007)

\begin{abstract}
Angular phase mixing in rapidly rotating or in strongly stratified flows is quantified for single-time single-point energy components, using linear theory. In addition to potential energy, turbulent kinetic energy is more easily analyzed in terms of its toroidal and poloidal components, and then in terms of vertical and horizontal components. Since the axial symmetry around the direction $\boldsymbol{n}$ (which bears both the system angular velocity and the mean density gradient) is consistent with basic dynamical equations, the input of initial anisotropy is investigated in the axisymmetric case. A general way to construct axisymmetric initial data is used, with a classical expansion in terms of scalar spherical harmonics for the 3D spectral density of kinetic energy $e$, and a modified expansion for the polarization anisotropy $Z$, which reflects the unbalance in terms of poloidal and toroidal energy components. The expansion involves Legendre polynomials of arbitrary order, $P_{2 n}^{0}(\cos \theta)$, $\left(n=0,1,2, \ldots, N_{0}\right)$, in which the term $[\cos \theta=(\boldsymbol{k} \cdot \boldsymbol{n}) /|\boldsymbol{k}|]$ characterizes the anisotropy in $\boldsymbol{k}$-wavespace; two sets of parameters, $\beta_{2 n}^{(e)}$ and $\beta_{2 n}^{(z)}$, separately generate the directional anisotropy and the polarization anisotropy. In the rotating case, the phase mixing results in damping the polarization anisotropy, so that toroidal and poloidal energy components asymptotically equilibrate after transient oscillations. Complete analytical solutions are found in terms of Bessel functions. The envelope of these oscillations decay with time like $(f t)^{-2}$ ( $f$ being the Coriolis parameter), whereas those for the vertical and horizontal components decay like $(f t)^{-3}$. The long-time limit of the ratio of horizontal component to vertical one depends only on $\beta_{2}^{(e)}$, which is eventually related to a classical component in structure-based modeling, independently of the degree of the expansion of the initial data. For the stratified case, both the degree of initial anisotropy and the initial unbalance in terms of potential and poloidal (or kinetic gravity wave) energy are investigated. The latter unbalance is characterized by a ratio $\chi / 2$, assuming initial proportionality between the kinetic energy spectrum and the potential energy one. The phase mixing yields asymptotic equipartition in terms of poloidal and potential energy components, and analytical solutions are found in terms of Weber functions. At large time, the damped oscillations for poloidal, potential and vertical components decay with time like $(N t)^{-1 / 2}$ ( $N$ is the buoyancy frequency), while the oscillations for the horizontal component decay with time like $(N t)^{-3 / 2}$. The long-time limit of the ratio of horizontal component to vertical one depends only on the parameters $\chi, \beta_{2}^{(e)}, \beta_{0}^{(z)}, \beta_{2}^{(z)}$, and $\beta_{4}^{(z)}$.

(C) 2007 American Institute of Physics. [DOI: 10.1063/1.2728934]
\end{abstract}

\section{INTRODUCTION}

The study of turbulence dynamics in a rotating stably stratified fluid presents interest in many geophysical or astrophysical applications. In the present study, we consider an initially anisotropic turbulence subjected to the Coriolis or the buoyancy forces. We use the linear theory to examine the role of the initial conditions in determining the subsequent time development of stratified or rotating flows. For this purpose, a systematic way is used here to generate anisotropic initial data for two-point single-time velocity correlations. Physically relevant initial data are chosen to be axisymmetric in agreement with the symmetry consistent with the basic dynamical equations. We use a modified spherical harmonic decomposition in terms of Legendre polynomials.
Wave modes, with their particular anisotropic dispersion relations, greatly complicate the dynamics of turbulence by permitting parametric instabilities and a richer range of possibilities for cascade dynamics, ranging from weak (waveturbulence) to fully developed strong turbulence. These possibilities are surveyed in the special case of gravity waves in a stratified nonrotating fluid. ${ }^{1}$

In the canonical case of a three-dimensional unbounded flow, with constant rotation parameter (Coriolis parameter $f$ ) and constant stratification parameter (Brunt-Vaisala frequency $N$ ), analytical solutions can be found for the initial value, or Cauchy problem, for the linearized Navier-Stokes equations with buoyancy forces in the Boussinesq approximation. Linear dynamics is characterized by superposed 
steady and unsteady motions, the first corresponding to quasigeostrophic (QG) motion and the second to dispersive inertia-gravity waves. The most general linear solution is generated by a Green's function in Fourier space, ${ }^{2,3}$ and this gives access to eigenmodes and to relevant dispersion laws of the wave motion. Related linear solutions can be derived for any statistical moments in terms of velocity and buoyancy fluctuations. The result is often known as rapid distortion theory (RDT), whose applications are essentially restricted to second order statistics.

On the one hand, purely linear dynamics yields marginal information if statistics are restricted to second order singletime correlations; there is no significant isotropy breaking since both total and QG energy are strictly conserved, keeping their three-dimensional spectral distribution constant in time. This disappointing behavior reflects the suppression of phase information by forming products of terms with the time dependence $e^{ \pm l \sigma t}, \sigma$ being the dispersion frequency of waves. An interesting behavior, however, was found at the level of two-time second order correlations ${ }^{2,4}$ with application to Lagrangian diffusivity, and at single-time third-order correlations, with application to nonlinear energy transfer and to wave-turbulence in the rotating ${ }^{5,6}$ and in the stratified ${ }^{7}$ flow case.

On the other hand, single-time second-order statistics are more informative if the RDT solution is started with anisotropic, or somewhat unbalanced (in terms of energy equipartition) initial data. The evolution continues to reflect the conservation of some energetic quantities (QG and total energy), but also exhibits trends towards equipartition via damped oscillations. These damped oscillations are found even in the purely inviscid case and reflect angular phase-mixing, which is directly controlled by the dispersion law of inertia-gravity waves. $^{2,4,5}$

The effect of angular phase-mixing can be understood from a simple integral

$$
I(f t)=\int_{0}^{1} \phi(x) \cos (2 x f t) d x
$$

which results in damped oscillations, developing in time $t$, and always reaches the zero value at large $t$ for any weighting function $\phi(x)$, up to regularity conditions. In the example above, $x f$ is the dispersion frequency of inertial waves, as investigated in Sec. IV. At a given value of $x$, the function in the integrand above exhibits pure oscillations in time, with period $\pi /(x f)$, but the period is continuously changed from $\pi / f$ to infinity in the integral; any discrete numerical scheme amounting to a finite $\Delta x$ can be questioned if the elapsed time is large enough. Correct decay of oscillations, even if satisfactorily captured by conventional DNS at short nondimensional time $f t$, can be wrongly recovered at larger $f t$. This issue yielded using the implicit numerical scheme given by the linear eigenmode decomposition for DNS of rapid rotation (e.g., Ref. 8). Accordingly, only analytical solutions are carried out in this paper. Our main objective is to predict time history for key quantities, with maximum accuracy, given an initial anisotropic input to linear theory.
Complex linear evolution, completely missed in conventional Reynolds stress modeling, was shown in the case of rapid rotation, with anisotropic initial data. 5 The rapid change (a quarter of a revolution) of the structure of the initial Reynolds stress tensor was interpreted as resulting from strict conservation of directional anisotropy and rapid damping of polarization anisotropy, justifying the splitting of the deviator $b_{i j}$ of the Reynolds stress tensor into two separate contributions, $b_{i j}^{(e)}$ and $b_{i j}^{(z)}$ (as also used in fully nonlinear studies ${ }^{6,8}$ ). This effect of damping polarization anisotropy reflects the above mentioned anisotropic phase mixing. This effect was recovered independently by Kassinos et al., ${ }^{10}$ and called "rotational randomization." The response of initially anisotropic flows to rotation was studied by Mansour et al. ${ }^{11}$ using both direct numerical simulation (DNS) and RDT with the same eigenmode decomposition (directional/polarization) as Cambon and Jacquin. ${ }^{6}$ Damped oscillations in RDT and DNS were shown to compare well. A pure return-to-isotropy, forced by rapid rotation, was also shown using some special version of a spectral model ${ }^{12}$ for initial data, as discussed in Sec. VI.

Similar comparisons between RDT and DNS were carried out by Refs. 13 and 14 and by Ref. 15, with identification of similar damped oscillations. In addition, the former authors have shown the importance of the initial ratio of kinetic energy to potential energy for determining the subsequent development of stratified turbulence.

The present study is along the line of the abovementioned RDT ones, considering physically relevant initial anisotropic data and analytically determining the development of energy components (such as the vertical contribution to kinetic energy), but we show as clearly as possible why solenoidal modes are useful in simplifying the description of the linear processes. The so-called Craya-Herring ${ }^{16}$ decomposition, with its counterpart in the complex plane ${ }^{5,17}$ (helical mode decomposition), is used for this purpose, and its close analogy with the poloidal/toroidal decomposition is exploited for physical interpretation in both flow cases (rotating and stratified). Eigenmode decomposition is simplified using this decomposition, which is moreover consistent with a rational anisotropic description of second order correlations, in terms of directional and polarization anisotropy. The way of building anisotropy can be related to the ansatz by Ref. 18, similarly to Ref. 19 , and more generally to the recent approach of Ref. 20.

The paper is organized as follows: In Sec. II, we introduce the linearized equations and we briefly discuss the condition for the validity of these equations. In Sec. III, the decomposition in terms of spherical harmonics to generate anisotropic initial data is introduced. In Sec. IV, we consider the case of solid body rotation, and we analytically determine the evolution of typical energy components, poloidal/ toroidal as well as horizontal/vertical. Long time behavior and asymptotic values of typical ratios are given, for initial data using arbitrary degrees of angular harmonics expansion. Stable stratification without rotation is addressed in Sec. V, using a similar analysis. In Sec. VI, some typical parameters for the initial data, which are called into play in the linear solutions, are physically interpreted and eventually expressed 
in terms of single-point deviatoric structure tensors. Section VII deals with our conclusion.

\section{GOVERNING EQUATIONS}

In this section, we introduce the linearized equations for the fluctuations of velocity and density, and we briefly discuss the condition for validity of these equations. The linear solutions for the Fourier components are given in a local frame attached to the wave vector, the Craya-Herring frame. ${ }^{16}$

\section{A. Linearized equations}

The governing dynamical equations for velocity $\boldsymbol{u}$ and buoyancy $b$ fluctuations are linearized Navier-Stokes equations within the Boussinesq approximation, or

$$
\frac{\partial \boldsymbol{u}}{\partial t}+f \boldsymbol{n} \times \boldsymbol{u}=\nabla \cdot p+b \boldsymbol{n}, \quad \frac{\partial b}{\partial t}=-N^{2} \boldsymbol{u} \cdot \boldsymbol{n} .
$$

Coriolis and buoyancy/stratification effects are given by constant $f$ (Coriolis parameter) and $N$ (Brunt-Waisala) frequencies, respectively. Here, $p$ is a modified pressure term only needed to ensure the solenoidal property $\nabla \cdot \boldsymbol{u}=0$, and $\boldsymbol{n}$ denotes the vertical upwards unit vector which bares both gravitational acceleration and system vorticity.

Conditions for validity of the linear theory were discussed as follows (e.g., Derbyshire and $\mathrm{Hunt}^{21}$ ): The above linearized equations are valid if the nonlinear term [i.e., $(\boldsymbol{u} \cdot \nabla) \boldsymbol{u}]$ in the Navier Stokes equations is small compared to either the Coriolis term $(\boldsymbol{n} \times \boldsymbol{u})$ or the buoyancy term $b \boldsymbol{n}$. At the same time, the term $(\boldsymbol{u} \cdot \boldsymbol{\nabla}) b$ must be small compared to $N^{2} \boldsymbol{n} \cdot \boldsymbol{u}$ in the equation for $b$, considering the nonstratified rotating case. At large time $(f t \gg 1)$, a condition for validity of (1) is that the Rossby number must be very small, i.e., $\left[R_{o t}=u /(f \ell) \ll 1\right]$ where $\ell$ is the eddy size and $u(\ell)$ is its characteristic velocity. Regarding the stratified nonrotating case, a condition for validity of (1) is that the turbulent Froude number must be very small, i.e., $F_{r t}=u /(N \ell) \ll 1$.

At low and moderate Reynolds number (as in laboratory experiments for grid turbulence and in most of the DNS), $R_{e}=u_{0} \ell_{0} / \nu$, where $\ell_{0}$ and $u_{0}$ are the length scale and the velocity scale of energy-containing eddies, $R_{o t} \sim R_{0}$ $=u_{0} /\left(f \ell_{0}\right) \ll 1 \quad\left[\right.$ respectively, $\left.F_{r t} \sim F_{r}=u_{0} /\left(N \ell_{0}\right) \ll 1\right]$ is the condition for the validity of (1) in the rotating (stratified) case since $u / \ell=\mathcal{O}\left(u_{0} / \ell_{0}\right)$. However, at high Reynolds number, $R_{o t} \sim R_{0}\left(\ell_{0} / \ell\right)^{2 / 3} \ll 1$ [respectively $\left.F_{r}\left(\ell_{0} / \ell\right)^{2 / 3} \ll 1\right]$ is the condition for validity of (1) in the rotating (stratified) case since $u / \ell=\mathcal{O}\left(\varepsilon^{1 / 3} \ell^{-2 / 3}\right)$, where $\varepsilon$ is the dissipation rate (e.g., Ref. 14).

The previous analysis must be compared to recent, weakly or not, theoretical nonlinear analyses, since the above-mentioned conditions of small Rossby and Froude numbers are not always sufficient and/or necessary. In rotating turbulence, nonlinearity is really weak in the limit of low Rossby number, but it can accumulate over a very large time in order to create a special cascade relevant for waveturbulence theory. Such a theory predicts a depletion of nonlinearity due to interacting dispersive inertial waves, so that a significant nonlinear cascade only appears at a very long time, typically ${ }^{6,22} f t \sim O\left(R_{o}^{-2}\right)$. Consequently, linear theory is relevant for a much larger elapsed time than what is suggested by conventional dimensional analysis in the rotating flow case. On the other hand, gravity waves dynamics only affect the poloidal part of the fluctuating motion in the stably stratified flow case, whereas the complementary part, or toroidal motion, remains essentially constant, as we will show again in the next section. Pure nonlinear interactions for the toroidal component are possibly important even in the low Froude number limit; additional depletion of nonlinearity possibly affects the interactions including waves only. Toroidal cascade was suggested to explain the horizontal layering of strongly stratified flows. ${ }^{3,7}$ Nevertheless, this is the linear phase-mixing of gravity waves which allows eventual emergence of the toroidal cascade, so that the linear limit remains useful to investigate. In short, basic linear time scales such as $N^{-1}$ or $f^{-1}$ characterize the period of rapid oscillations, but more relevant and larger time scales characterize the phasemixing which controls the decay of the envelopes of rapid oscillations. Finally, nonlinear time scales could be evaluated, but $l / u$ is only a crude a priori estimate, and refined nonlinear theories are needed. In wave turbulence, the nonlinear time scale is very large, and the one of the toroidal cascades in the stably stratified case cannot be a priori evaluated.

Finally, in the absence of effects of mean shear or mean strain, there is no space distortion, whereas interacting dispersive waves can induce a depletion of nonlinearity and delay it. Accordingly, it is relevant to use linear theory for large times, and the acronym RDT will be avoided from now on, except for some initialization processes.

\section{B. Linear solutions for an optimal set of fluctuating variables}

The solutions of these equations are most easily obtained via Fourier synthesis, $\quad[\boldsymbol{u}(\boldsymbol{x}, t), b(\boldsymbol{x}, t)]$ $=\Sigma_{k}[\hat{\boldsymbol{u}}(\boldsymbol{k}, t), \hat{b}(\boldsymbol{k}, t)] \exp (\boldsymbol{k} \cdot \boldsymbol{x})$, where $\boldsymbol{k}$ is the wave vector, the discrete summation possibly being replaced by an integral. A spectral formalism allows us to take into account the solenoidal condition, which amounts to the orthogonality of $\hat{\boldsymbol{u}}$ with $\boldsymbol{k}$. The latter simple condition then yields a reduced number of components by projecting $\boldsymbol{u}$ in the Craya-Herring ${ }^{16}$ frame. This frame coincides with the horizontal fixed frame if $\boldsymbol{k}$ is vertical $(\boldsymbol{k} \| \boldsymbol{n})$, and otherwise is defined by

$$
\mathrm{e}^{(1)}=\frac{\boldsymbol{k} \times \boldsymbol{n}}{|\boldsymbol{k} \times \boldsymbol{n}|}, \quad \mathrm{e}^{(2)}=\frac{\boldsymbol{k} \times(\boldsymbol{k} \times \boldsymbol{n})}{|\boldsymbol{k} \times \boldsymbol{n}|}
$$

so that

$$
\hat{\boldsymbol{u}}=u^{(1)} \mathbf{e}^{(1)}+u^{(2)} \mathbf{e}^{(2)}
$$

(see Ref. 3 for details). If $k_{\perp}=|\boldsymbol{k} \times \boldsymbol{n}| \neq 0, u^{(1)}$ and $u^{(2)}$ correspond to toroidal and poloidal velocity components in physical space. The limit of pure vertical wave numbers gives the vertically sheared horizontal flow (VSHF) (Ref. 23), a particular mode of motion which is very important in the stratified flow case. For convenience, a third component $u^{(3)}$ is related to the buoyancy force, using the scaling 


$$
u^{(3)}=-\frac{\hat{b}}{N}
$$

which ensure that $\left\langle u^{(3) *} u^{(3)}\right\rangle / 2$ is the spectral density of potential energy, as $\left\langle\hat{u}_{i}^{*} \hat{u}_{i}\right\rangle / 2$ is the spectral density of kinetic energy, in the stratified case. Here, $\langle\cdot\rangle$ denotes ensemble averaging and the superscript $*$ denotes complex conjugate. If $N=0$ (pure rotating unstratified case), this third component is simply ignored. The general problem in five components in physical space $\left(u_{1}, u_{2}, u_{3}, p, b\right)$ therefore reduces to a threecomponent $\left(u^{(1)}, u^{(2)}, u^{(3)}\right)$ one, and the system of Eq. (1) becomes

$$
\frac{\partial}{\partial t}\left(\begin{array}{l}
u^{(1)} \\
u^{(2)} \\
u^{(3)}
\end{array}\right)+\left(\begin{array}{ccc}
0 & -\sigma_{r} & 0 \\
\sigma_{r} & 0 & -\sigma_{s} \\
0 & \sigma_{s} & 0
\end{array}\right)\left(\begin{array}{l}
u^{(1)} \\
u^{(2)} \\
u^{(3)}
\end{array}\right)=0
$$

in which

$$
\begin{aligned}
& \sigma_{r}=f \frac{k_{\|}}{k}=f \frac{\boldsymbol{k} \cdot \boldsymbol{n}}{k}=f \cos \theta, \\
& \sigma_{s}=N \frac{k_{\perp}}{k}=N \frac{|\boldsymbol{k} \times \boldsymbol{n}|}{k}=N \sin \theta
\end{aligned}
$$

are the dispersion frequency of inertial and gravity waves, respectively. It clearly appears that the anisotropic distribution is characterized by the polar angle $\theta=(\hat{\boldsymbol{k}, \boldsymbol{n}})$. The solution of (5) is of the form

$$
\left(\begin{array}{l}
u^{(3)} \\
u^{(2)} \\
u^{(1)}
\end{array}\right)=\left(\begin{array}{ccc}
\cos \sigma_{s} t & \sin \sigma_{s} t & 0 \\
-\sin \sigma_{s} t & \cos \sigma_{s} t & 0 \\
0 & 0 & 1
\end{array}\right)\left(\begin{array}{c}
u_{0}^{(3)} \\
u_{0}^{(2)} \\
u_{0}^{(1)}
\end{array}\right)
$$

for the stratified case, and

$$
\left(\begin{array}{l}
u^{(1)} \\
u^{(2)} \\
u^{(3)}
\end{array}\right)=\left(\begin{array}{ccc}
\cos \sigma_{r} t & \sin \sigma_{r} t & 0 \\
-\sin \sigma_{r} t & \cos \sigma_{r} t & 0 \\
0 & 0 & 1
\end{array}\right)\left(\begin{array}{c}
u_{0}^{(1)} \\
u_{0}^{(2)} \\
u_{0}^{(3)}
\end{array}\right)
$$

for the rotating case. Here, the subscript 0 denotes the initial values. As it can be expected, the above solutions are similar, but the toroidal mode $\left[u^{(1)}\right]$ and the potential mode $\left[u^{(3)}\right]$ in the stratified case are interchanged with respect to the rotating case. Incidentally, in the stratified case only, poloidal and toroidal exactly correspond to the "wave" and "vortex" modes used by Riley et $a l^{24}$

\section{INITIALLY AXISYMMETRIC TURBULENCE}

\section{A. Statistical descriptors: Arbitrary anisotropy}

We recall a general formulation ${ }^{5}$ of the spectral tensor of second order velocity correlations $\hat{R}_{i j}=\mathfrak{R}\left\langle\hat{u}_{i}^{*} \hat{u}_{j}\right\rangle$, which is valid for arbitrary anisotropy,

$$
\hat{R}_{i j}=\underbrace{\frac{E(k, t)}{4 \pi k^{2}} P_{i j}}_{\text {Isotropic part }}+\underbrace{\mathcal{E}(\boldsymbol{k}, t) P_{i j}}_{\text {Directional anisotropy }}+\underbrace{\mathfrak{R}\left(Z(\boldsymbol{k}, t) N_{i} N_{j}\right)}_{\text {Polarization anisotropy }}
$$

in which $P_{i j}=e_{i}^{(1)} e_{j}^{(1)}+e_{i}^{(2)} e_{j}^{(2)}=\delta_{i j}-k_{i} k_{j} / k^{2}$ is the conventional transverse projection operator and $N_{i}=e_{i}^{(2)}-l e_{i}^{(1)}$ is a complex "helical mode," $E(k, t)$ is the kinetic energy radial spectrum (i.e., the spherical average)

$E(k, t)=\frac{1}{2} \int_{S_{k}} \hat{R}_{i i}(\boldsymbol{k}, t) d^{2} \boldsymbol{k}, \quad \mathcal{E}(\boldsymbol{k}, t)=\frac{1}{2} \hat{R}_{i i}(\boldsymbol{k}, t)-\frac{E(k, t)}{4 \pi k^{2}}$

and

$$
\begin{aligned}
Z(k, t) & =\frac{1}{2} N_{i}^{*} \hat{R}_{i j}(\boldsymbol{k}, t) N_{j}^{*} \\
& =\frac{1}{2}\left(\left\langle u^{(2)} u^{(2)^{*}}\right\rangle-\left\langle u^{(1)} u^{(1)^{*}}\right\rangle\right)+\imath \Re\left\langle u^{(1)} u^{(2) *}\right\rangle
\end{aligned}
$$

is a complex-valued deviatoric term. Directional anisotropy, in close connection with dimensionality, indicates the departure of the distribution of spectral 3D energy density $e$ $=(1 / 2) \hat{R}_{i i}(\boldsymbol{k}, t)$ from its spherical equipartition $E(k, t) / 4 \pi k^{2}$, whereas the polarization anisotropy reflects that the directions of $\hat{\boldsymbol{u}}$, at fixed $\boldsymbol{k}$ and located in the plane normal to $\boldsymbol{k}$, are not statistically equivalent when rotating around $\boldsymbol{k}$. The real part of $Z$ also reflects the unbalance between toroidal and poloidal kinetic energy density. The imaginary part of $Z$, as a mixed poloidal/toroidal co-spectrum, also reflects the breaking of mirror symmetry, and can be linked to the "stropholysis" term ${ }^{10}$ when rapid rotation is present.

\section{B. Decomposition in terms of spherical harmonics}

Restricted to axial symmetry (around $\boldsymbol{n}$ ), which is the simplest symmetry consistent with the governing equations, we find

$$
\begin{aligned}
& \mathcal{E}(\boldsymbol{k}, t)=\frac{E(k, t)}{4 \pi k^{2}}\left[\sum_{n=1}^{N_{0}} e_{2 n}(k, t) P_{2 n}^{0}(\cos \theta)\right], \\
& Z(\boldsymbol{k}, t)=\sin ^{2} \theta \frac{E(k, t)}{4 \pi k^{2}}\left[\sum_{m=0}^{2 N_{0}} z_{m}(k, t) P_{m}^{0}(\cos \theta)\right]
\end{aligned}
$$

using a modified spherical harmonic decomposition in terms of Legendre polynomials $P_{m}^{0}$. The polar angle $\theta$ characterizes the anisotropy, with

$$
k_{\perp}=k \sin \theta=k \sqrt{1-x^{2}}, \quad k_{\|}=k \cos \theta=k x .
$$

As an important remark, this decomposition, suggested by previous studies in Cambon's team from Ref. 19 does not apply in the same way to the "directional" $\mathcal{E}$ and to the "polarization" part $Z$ of the anisotropy. The weighting factor $\sin ^{2} \theta$ allows a correct convergence of the polarization $Z$ towards 0 when the wave vector becomes aligned with the axial direction. This approach is consistent with the ansatz ${ }^{18}$ for axisymmetric turbulence, choosing only even degrees $m$ $=2 n$. In addition, the "polarization" part $Z$ is chosen to be real in agreement with mirror symmetry (also assumed by Ref. 18). More generally, for arbitrary anisotropy, the use of conventional scalar spherical harmonics for "directional," but not for "polarization," anisotropy reflects the need for a more accurate approximation using higher order expansions based on irreducible representations of the rotation group $S O^{3}$. This representation theory has recently been found to be 
very useful in clarifying the scaling properties of correlation functions in turbulent flows. ${ }^{25}$ On the other hand, some strongly anisotropic "two-component" initial data used in RDT (Ref. 26) correspond to $\Phi^{1}(k)=E /\left(4 \pi k^{2}\right)+Z(k)=0$ and $\Phi^{2}(k)=E /\left(4 \pi k^{2}\right)-Z(k) \neq 0$; they are not only physically questionable but mathematically wrong.

\section{ROTATING HOMOGENEOUS TURBULENCE}

We determine the evolution of poloidal, toroidal, vertical, and horizontal components in terms of the Bessel functions. We show that the ratio $\mathcal{K}^{(\mathrm{pol})} / \mathcal{K}^{\text {(tor) }}$ exactly equilibrates after damped oscillations, independently of the degree of the initial angular harmonic expansion, and the long-time limit of the ratio $\mathcal{K}^{\text {(hor) }} / \mathcal{K}^{\text {(ver) }}$ depends only on the initial directional anisotropy, after damping of the initial polarization anisotropy.

\section{A. Spectral densities of energies}

In the rotating case, with $N=0, u^{(3)}$ is irrelevant, as is the potential energy. From the solution (9) we easily show that the radial spectrum and the directional anisotropy do not change with time, i.e.,

$$
E(k, t)=E(k, 0), \quad \mathcal{E}(\boldsymbol{k}, t)=\mathcal{E}(\boldsymbol{k}, 0),
$$

whereas the polarization anisotropy exhibits an oscillatory behavior if its initial value is not zero,

$$
Z(\boldsymbol{k}, t)=Z(\boldsymbol{k}, 0) e^{2 \imath f t x}=Z(k, x, 0) \cos (2 f t x) .
$$

By substituting (16) and (17) into (10), we obtain the spectral density of the vertical component as,

$$
\begin{aligned}
\frac{1}{2}\left\langle\hat{u}_{3}(t) \hat{u}_{3}^{*}(t)\right\rangle= & \frac{1}{2}\left(1-x^{2}\right)\left(\frac{E(k, 0)}{4 \pi k^{2}}+\mathcal{E}(k, x, 0)\right. \\
& +Z(k, x, 0) \cos (2 f t x)) .
\end{aligned}
$$

In agreement with $\hat{u}_{i} n_{i}=-\sin \theta u^{(2)}$ using (3), the spectral density of poloidal contribution to kinetic energy takes the form,

$$
\begin{aligned}
\frac{1}{2}\left\langle u^{(2)}(t) u^{(2) *}(t)\right\rangle= & \frac{1}{2}\left(\frac{E(k, 0)}{4 \pi k^{2}}+\mathcal{E}(k, x, 0)\right. \\
& +Z(k, x, 0) \cos (2 f t x)) .
\end{aligned}
$$

Because the spectral density of kinetic energy is conserved in the linear inviscid limit, the poloidal component is then derived from the latter equation,

$$
\begin{aligned}
\frac{1}{2}\left\langle u^{(1)}(t) u^{(1) *}(t)\right\rangle= & \frac{1}{2}\left(\frac{E(k, 0)}{4 \pi k^{2}}+\mathcal{E}(k, x, 0)\right. \\
& -Z(k, x, 0) \cos (2 f t x)),
\end{aligned}
$$

while the horizontal one is derived by substracting (18) from (16),

$$
\begin{aligned}
& \frac{1}{4}\left[\left\langle\hat{u}_{1}(t) \hat{u}_{1}^{*}(t)\right\rangle+\left\langle\hat{u}_{2}(t) \hat{u}_{2}^{*}(t)\right\rangle\right] \\
& \quad=\frac{1}{2}\left(1-x^{2}\right)\left(\frac{E(k, 0)}{4 \pi k^{2}}+\mathcal{E}(k, x, 0)-Z(k, x, 0) \cos (2 f t x)\right) .
\end{aligned}
$$

\section{B. Poloidal and toroidal parts of kinetic energy}

By substituting the expressions of $\mathcal{E}(k, x, 0)$ and $Z(k, x, 0)$, given by (16) and (17), into (19) and then integrating it over wave space we obtain the poloidal energy component as

$$
\frac{2 \mathcal{K}^{(\mathrm{pol})}(t)}{\mathcal{K}(0)}=1+\epsilon \frac{15}{8} \sum_{n=0}^{N_{0}} \beta_{2 n}^{(z)} \int_{-1}^{+1}\left(1-x^{2}\right) P_{2 n}^{0}(x) \cos (2 f t x) d x
$$

Using the fact that the polynomials $P_{0}^{0}(x), P_{2}^{0}(x), P_{4}^{0}(x), \ldots$ are orthogonal, ${ }^{27}$ i.e.,

$$
\int_{-1}^{+1} P_{n}^{0}(x) P_{m}^{0}(x) d x=\left\{\begin{array}{cc}
0 & \text { if } m \neq n \\
\frac{2}{2 n+1} & \text { if } m=n
\end{array}\right.
$$

and they can be expanded as (see the Appendix)

$$
P_{2 n}^{0}(x)=\sum_{\ell=0}^{n} a_{\ell, n}\left(1-x^{2}\right)^{\ell},
$$

where $a_{0, n}=1$, we obtain

$$
\begin{aligned}
\frac{2 \mathcal{K}^{(\mathrm{pol})}(t)}{\mathcal{K}(0)}= & 1+\epsilon \frac{15 \sqrt{\pi}}{8} \sum_{n=0}^{N_{0}} \sum_{\ell=0}^{n} \beta_{2 n}^{(z)} a_{\ell, n}(\ell+1) !(f t)^{-(\ell+3 / 2)} \\
& \times J_{\ell+3 / 2}(2 f t)
\end{aligned}
$$

in which $\epsilon=1$ and $J_{\ell+3 / 2}(z)$ is the Bessel function of the first kind of order $\ell+3 / 2$ (see Ref. 27). For example, $J_{3 / 2}(z)$ $=\sqrt{2 / \pi z}(\sin z / z-\cos z)$. Here, the parameters

$$
\begin{aligned}
& \beta_{2 n}^{(e)}=-\frac{1}{15 \mathcal{K}(0)} \int_{0}^{\infty} E(k, 0) e_{2 n}(k) d k, \\
& \beta_{2 n}^{(z)}=\frac{4}{15 \mathcal{K}(0)} \int_{0}^{\infty} E(k, 0) z_{2 n}(k) d k
\end{aligned}
$$

characterize the directional anisotropy and the polarization anisotropy, respectively (the special prefactor $1 / 15$ is justified in Sec. VI), and $\mathcal{K}(0)=\int_{0}^{\infty} E(k, 0) d k$ is the initial kinetic energy. The toroidal contribution to kinetic energy is obtained by replacing in (24) $\epsilon=1$ by $\epsilon=-1$. Both short-time and long-time evolution can be derived from the latter expressions. Short-time behavior $(f t \ll 1)$ is obtained by using the series representation of $J_{\nu}(z)$ (see Ref. 27), and yields 


$$
\begin{aligned}
\frac{2 \mathcal{K}^{(\mathrm{pol})}(t)}{\mathcal{K}(0)}= & 1+\frac{15 \sqrt{\pi}}{8} \sum_{n=0}^{N_{0}} \sum_{\ell=0}^{n} \beta_{2 n}^{(\mathrm{z})} a_{\ell, n}(\ell+1) !\left[\frac{1}{\Gamma(\ell+5 / 2)}\right. \\
& \left.-\frac{1}{\Gamma(\ell+7 / 2)}(f t)^{2}+\ldots\right] .
\end{aligned}
$$

During the initial phase and when

$$
\sum_{n=0}^{N_{0}}\left[\sum_{\ell=0}^{n} \frac{a_{\ell, n}(\ell+1) !}{\Gamma(\ell+5 / 2)}\right] \beta_{2 n}^{(z)}>0
$$

the toroidal contribution of kinetic energy grows with time [like $\left.(f t)^{2}\right]$, whereas the poloidal contribution decays with time [like $(f t)^{2}$ ]. Later, the energy is exchanged periodically between these components. At large time $(f t \gg 1)$, the Bessel function $J_{\ell+3 / 2}(2 f t)$ behaves as (see Ref. 27),

$$
J_{\ell+3 / 2}(2 f t) \sim \frac{1}{\sqrt{\pi f t}} \cos \left(2 f t-\frac{\ell \pi}{2}\right) .
$$

Therefore, the contribution of the first spherical harmonics $z_{0}(k)$ on the evolution of these components is important since the oscillations decay with time like $(f t)^{-2}$. In addition, these components exactly equilibrate after damped oscillations, $\left(\mathcal{K}^{\text {(tor) }} / \mathcal{K}^{(\mathrm{pol})}\right)_{\infty}=1$, independently of the initial angular harmonic expansion.

\section{Vertical and horizontal parts of kinetic energy}

In a similar way, we determine both the vertical and horizontal parts of kinetic energy,

$$
\begin{aligned}
\frac{3 \mathcal{K}^{(\mathrm{ver})}(t)}{\mathcal{K}(0)}= & 1+3 \beta_{2}^{(e)}+\frac{45 \sqrt{\pi}}{16} \sum_{n=0}^{N_{0}} \sum_{\ell=0}^{n} \beta_{2 n}^{(z)} \\
& \times a_{\ell, n}(\ell+2) !(f t)^{-(\ell+5 / 2)} J_{\ell+5 / 2}(2 f t), \\
\frac{3 \mathcal{K}^{(\text {hor })}(t)}{\mathcal{K}(0)}= & 1-\frac{3}{2} \beta_{2}^{(e)}-\frac{45 \sqrt{\pi}}{32} \sum_{n=0}^{N_{0}} \sum_{\ell=0}^{n} \beta_{2 n}^{(z)} \\
& \times a_{\ell, n}(\ell+2) !(f t)^{-(\ell+5 / 2)} J_{\ell+5 / 2}(2 f t) .
\end{aligned}
$$

When $f t \ll 1$, both vertical and horizontal parts of kinetic energy behave like $(f t)^{2}$. At large time $(f t \gg 1)$, the oscillations for these parts of kinetic energy decrease like $t^{-3}$. In addition, the ratio of horizontal to vertical energy components reaches an asymptotic value, or

$$
\eta(t)=\mathcal{K}^{(\text {hor })}(t) / \mathcal{K}^{(\text {ver })}(t) \rightarrow 1-\frac{9 \beta_{2}^{(e)}}{2+6 \beta_{2}^{(e)}}
$$

as $f t \rightarrow \infty$ independently of the degree of the initial angular harmonic expansion. This is rediscussed in Sec. VI.

Viscous effects can easily be added, since the initial spectrum $E(k)$ has just to be replaced by $E(k) \exp \left(-2 \nu k^{2} t\right)$. In the above equation, $\beta_{2}^{(e)}$ could be replaced by its counterpart $\beta_{(\mathrm{vis}) 2}^{(e)}(t)$, in the limit of large times, as discussed further in Sec. V D.

\section{STABLY STRATIFIED HOMOGENEOUS TURBULENCE}

In this section, we use the solution (8) to express the spectral density of energy in terms of the scalars $\mathcal{E}(\boldsymbol{k}, 0)$ and $Z(\boldsymbol{k}, 0)$. Considering initial axisymmetric data generated by $\mathcal{E}(k, x, 0)$ and $Z(k, x, 0)$ given by Eqs. (13) and (14), the spectral density of energy is integrated over wave space to obtain the time development of typical energy components, poloidal/toroidal, horizontal/vertical, and potential. Both short-time and long-time behaviors of energies are addressed. We show that the poloidal part of the kinetic energy and the potential energy exactly equilibrate after damped oscillations, independently of the degree of the initial angular harmonic expansion. The long-time limit of the ratio of the vertical part to the horizontal one is, however, sensitive to the initial anisotropic data.

\section{A. Spectral density of energy}

In the stratified case, the linear inviscid solution [Eq. (8)] yields

$$
\begin{aligned}
& \left\langle u^{(1)}(t) u^{(1)^{*}}(t)\right\rangle=\left\langle u_{0}^{(1)} u_{0}^{(1)^{*}}\right\rangle, \\
& \left\langle u^{(2)}(t) u^{(2)^{*}}(t)\right\rangle+\left\langle u^{(3)}(t) u^{(3) *}(t)\right\rangle=\left\langle u_{0}^{(2)} u_{0}^{(2) *}\right\rangle+\left\langle u_{0}^{(3)} u_{0}^{(3) *}\right\rangle,
\end{aligned}
$$

meaning that the spectral density of the toroidal contribution to kinetic energy is strictly conserved. Also the sum of poloidal and potential spectral energy densities, which corresponds to total gravity wave energy, is conserved.

In the present study, we assume that the initial poloidal buoyancy flux is zero, or $\Re\left\langle u_{0}^{(2)} u_{0}^{(3)^{*}}\right\rangle=0$ (the other component, or toroidal density flux is always zero in the axisymmetric case with mirror symmetry ${ }^{7}$ ), and that the spectral density of potential energy is proportional to the spectral density of kinetic energy, i.e.,

$$
\begin{aligned}
\left\langle u_{0}^{(3)} u_{0}^{(3)^{*}}\right\rangle & =\frac{\chi}{2}\left(\left\langle u_{0}^{(1)} u_{0}^{(1)^{*}}\right\rangle+\left\langle u_{0}^{(2)} u_{0}^{(2) *}\right\rangle\right) \\
& =\frac{\chi}{2}\left(\frac{E(k, 0)}{2 \pi k^{2}}+2 \mathcal{E}(k, x, 0)\right),
\end{aligned}
$$

where $\chi$ is a constant. The latter assumption leads to the relationship $E^{\text {(pot) }}(k, 0)=(\chi / 2) E(k, 0)$ used in several studies (e.g., Ref. 14). In view of (32) and the following relationship:

$$
\left\langle u_{0}^{(2)} u_{0}^{(2) *}\right\rangle=\frac{E(k, 0)}{4 \pi k^{2}}+\mathcal{E}(k, x, 0)+Z(k, x, 0),
$$

deduced from (10), we express the spectral density of the poloidal component 


$$
\begin{aligned}
\frac{1}{2}\left\langle u^{(2)}(t) u^{(2)^{*}}(t)\right\rangle= & \frac{1}{4}\left(\left\langle u_{0}^{(3)} u_{0}^{(3) *}\right\rangle+\left\langle u_{0}^{(2)} u_{0}^{(2) *}\right\rangle\right) \\
& -\frac{1}{4}\left(\left\langle u_{0}^{(3)} u_{0}^{(3) *}\right\rangle-\left\langle u_{0}^{(2)} u_{0}^{(2) *}\right\rangle\right) \cos 2 \sigma_{s} t,
\end{aligned}
$$

in terms of the scalars $E(k, 0), \mathcal{E}(k, x, 0)$, and $Z(k, x, 0)$,

$$
\begin{aligned}
\frac{1}{2}\left\langle u^{(2)}(t) u^{(2)^{*}}(t)\right\rangle \\
=\frac{1}{4}\left[(\chi+1)\left(\frac{E(k, 0)}{4 \pi k^{2}}+\mathcal{E}(k, x, 0)\right)+Z(k, x, 0)\right] \\
-\frac{\epsilon}{4}\left[(\chi-1)\left(\frac{E(k, 0)}{4 \pi k^{2}}+\mathcal{E}(k, x, 0)\right)-Z(k, x, 0)\right] \\
\quad \times \cos (2 N t x),
\end{aligned}
$$

where $\epsilon=1$. The expression of the spectral density of potential energy is also given by (35) with $\epsilon=-1$. As for the expression of the spectral density of the vertical part, it is obtained by multiplying the rhs of $(35)$ by $\left(1-x^{2}\right)$.

By substituting the expression of $\mathcal{E}(k, x, 0)$ and $Z(k, x, 0)$ given by (13) and (14), respectively, into (35) we obtain

$$
\begin{aligned}
\frac{1}{2}\left\langle u^{(2)}(t) u^{(2)^{*}}(t)\right\rangle \\
=\left[\frac{(\chi+1)}{4} \frac{E(k, 0)}{4 \pi k^{2}}+\frac{(\chi+1)}{4} \sum_{n=1}^{N_{0}} \frac{E(k, 0)}{4 \pi k^{2}} e_{2 n}(k) P_{2 n}(x)\right. \\
\left.+\frac{1}{4} \sum_{n=0}^{N_{0}} \frac{E(k, 0)}{4 \pi k^{2}} z_{2 n}(k) P_{2 n}(x)\right]+\cos \left(2 N t \sqrt{1-x^{2}}\right) \\
\times\left[\frac{(\chi-1)}{4} \frac{E(k, 0)}{4 \pi k^{2}}+\frac{(\chi-1)}{4} \sum_{n=1}^{N_{0}} \frac{E(k, 0)}{4 \pi k^{2}} e_{2 n}(k) P_{2 n}(x)\right.
\end{aligned}
$$

$$
\left.-\frac{1}{4} \sum_{n=0}^{N_{0}} \frac{E(k, 0)}{4 \pi k^{2}} z_{2 n}(k) P_{2 n}(x)\right]
$$

The above spectral density is integrated over wave space to obtain the development of poloidal contribution to kinetic energy.

\section{B. Poloidal part of kinetic energy and potential energy}

The integration of (36) over wave space yields

$$
\begin{aligned}
\frac{2 \mathcal{K}^{(\mathrm{pol})}(t)}{\mathcal{K}(0)}= & {\left[\frac{(\chi+1)}{2}+\frac{5}{4} \beta_{0}^{(z)}-\frac{1}{2} \beta_{2}^{(z)}-\frac{(\chi-1)}{4} I_{0}(2 N t)\right] } \\
& +\left[\frac{15(\chi-1)}{4} \sum_{n=1}^{N_{0}} \sum_{\ell=0}^{n} \beta_{2 n}^{(e)} a_{\ell, n} I_{\ell}(2 N t)\right] \\
& +\left[\frac{15}{16} \sum_{n=0}^{N_{0}} \sum_{\ell=0}^{n} \beta_{2 n}^{(z)} a_{\ell, n} I_{\ell+1}(2 N t)\right]
\end{aligned}
$$

where

$$
I_{n}(2 N t)=\int_{-1}^{+1}\left(1-x^{2}\right)^{n} \cos \left(2 N t \sqrt{1-x^{2}}\right) d x .
$$

Furthermore, the latter integral can be expressed in terms of the Weber functions (see the Appendix), which are associated with the Bessel functions ${ }^{27,28}$

$I_{\ell-1}(2 N t)=\frac{\pi}{2^{2 \ell-2}} \sum_{m=0}^{\ell-1} \frac{(-1)^{(\ell+m-1)}(2 \ell-1) !}{m !(2 \ell-1+m) !} W_{2 \ell-2 m-1}(2 N t)$.

Consequently, the poloidal contribution to kinetic energy takes the form

$$
\begin{aligned}
\frac{2 \mathcal{K}^{(\mathrm{pol})}(t)}{\mathcal{K}(0)}= & {\left[\frac{(\chi+1)}{2}+\frac{5}{4} \beta_{0}^{(z)}-\frac{1}{2} \beta_{2}^{(z)}-\epsilon \frac{(\chi-1)}{4} \pi W_{1}\right] } \\
& +\epsilon\left[\frac{15(\chi-1)}{4} \sum_{n=1}^{N_{0}} \sum_{\ell=1}^{n+1} \sum_{m=0}^{\ell-1}\left(\beta_{2 n}^{(e)} \frac{\pi}{2^{2 \ell-2}} a_{\ell-1, n}\right) \frac{(-1)^{(\ell+m-1)}(2 \ell-1) !}{m !(2 \ell-1-m) !} W_{2 \ell-2 m-1}\right] \\
& +\epsilon\left[\frac{15}{16} \sum_{n=0}^{N_{0}} \sum_{\ell=2}^{n+2} \sum_{m=0}^{\ell-1}\left(\beta_{2 n}^{(z)} \frac{\pi}{2^{2 \ell-2}} a_{\ell-2, n}\right) \frac{(-1)^{(\ell+m-1)}(2 \ell-1) !}{m !(2 \ell-1-m) !} W_{2 \ell-2 m-1}\right],
\end{aligned}
$$

with $\epsilon=1$. The potential energy, $\mathcal{K}^{\text {(pot) }}$, is obtained by replacing in the above expression $\epsilon=1$ by $\epsilon=-1$. As for the vertical density flux, $\left\langle u_{3} b\right\rangle$, can be determined using the following relation:

$$
\frac{d}{d t} \mathcal{K}^{(\mathrm{pot})}=-\left\langle u_{3} b\right\rangle
$$

derived from Eq. (1), and the functional relation $2 d W_{n} / d t=W_{n-1}-W_{n+1}$.

For initial isotropic data [i.e., $\left.\beta_{2 n}^{(z)}=\beta_{2 n}^{(e)}=0\right]$, these energies reduce to 


$$
\begin{aligned}
& \frac{2 \mathcal{K}^{(\mathrm{pol})}(t)}{\mathcal{K}(0)}=\frac{(\chi+1)}{2}-\frac{(\chi-1)}{4} \pi W_{1}(2 N t), \\
& \frac{2 \mathcal{K}^{(\mathrm{pot})}(t)}{\mathcal{K}(0)}=\frac{(\chi+1)}{2}+\frac{(\chi-1)}{4} \pi W_{1}(2 N t),
\end{aligned}
$$

indicating that, at $\chi=1$, there is no linear evolution; this case was studied in Ref. 7 for emphasizing nonlinear effects, whereas the peculiar RDT solution for this flow case was also quoted by Hanazaki and Hunt. ${ }^{14}$ The evolution of $\mathcal{K}^{(\mathrm{pol})}$, $\mathcal{K}^{\text {(pot) }}$ and $\mathcal{K}^{\text {(tor) }}(=$ constant $)$, normalized by $\mathcal{K}^{\text {(ver) }}(0)$, is shown in Fig. 1(a) for isotropic initial data with $\chi=0,1,2$.

Both short-time and long-time evolution can be derived from (40). Short-time behavior $(N t \ll 1)$ is obtained by using the series representation of $W_{2 n+1}(2 N t)$ (see Ref. 27)

$$
W_{2 n+1}(2 N t)=\sum_{\ell=0}^{\infty} \frac{(-1)^{\ell+n}(N t)^{2 \ell}}{\Gamma(\ell+n+3 / 2) \Gamma(\ell-n+1 / 2)},
$$

where $\Gamma(z)$ is the Gamma function ${ }^{27}$

$\Gamma\left(m+\frac{1}{2}\right)=\frac{\sqrt{\pi}}{2^{m}}(2 m-1) ! !, \quad \Gamma\left(\frac{1}{2}-m\right)=(-1)^{m} \frac{2^{m} \sqrt{\pi}}{(2 m-1) ! !}$.

During the initial phase, both $\mathcal{K}^{(\mathrm{pol})}$ and $\mathcal{K}^{(\mathrm{pot})}$ behave like $(N t)^{2}$, and if $\mathcal{K}^{(\mathrm{pol})}$ decreases with time, then $\mathcal{K}^{\text {(pot) }}$ increases and vice versa, since, in the linear inviscid limit, the sum of these parts of energy is strictly conserved, as the toroidal part. Later, the energy is exchanged periodically between the poloidal kinetic energy and the potential energy. At large time $(N t \gg 1)$, the Weber function $W(2 N t)$ behaves like the Bessel function of the second kind $U(2 N t)$ (also called the Neumann function), so that

$$
W_{n}(2 N t) \sim-\frac{1}{\sqrt{\pi N t}} \sin \left(2 N t-\frac{n \pi}{2}-\frac{\pi}{4}\right) .
$$

Accordingly, The oscillations for $\mathcal{K}^{(\mathrm{pol})}$ and $\mathcal{K}^{(\mathrm{pot})}$ decay with time proportionally to $t^{-1 / 2}$, and the oscillation period asymptotically approaches $T=\pi / N$ at long times. Because $W_{n}(2 N t) \rightarrow 0$ as $N t \rightarrow \infty$, the long-time limit of these energy components depends only on $\beta_{0}^{(z)}$ and $\beta_{2}^{(z)}$, and not on $\beta_{2 n}^{(e)}$.
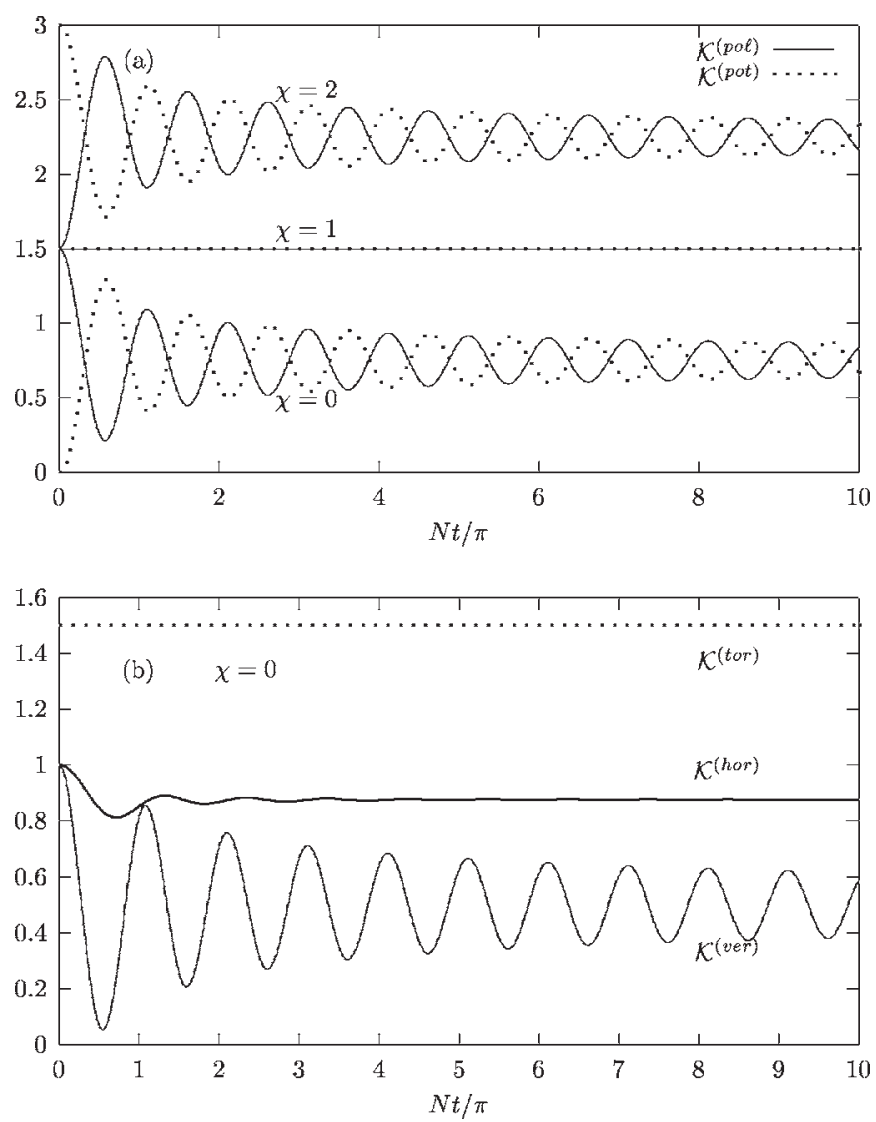

FIG. 1. Stable stratification, isotropic initial data with initially unbalanced poloidal/potential energy. The toroidal component remains constant and equal to the initial poloidal component, Eqs. (42) and (43).

Furthermore, they exactly equilibrate after damped oscillations, i.e., $\left(\mathcal{K}^{(\mathrm{pot})} / \mathcal{K}^{(\mathrm{pol})}\right)_{\infty}=1$, independently on the degree of the initial angular harmonic expansion.

\section{Vertical and horizontal contributions to kinetic energy}

The spectral density of vertical kinetic energy is derived by multiplying the rhs of (34) by $\left(1-x^{2}\right)$, as already indicated. The integration of $\left\langle u_{3} u_{3}^{*}\right\rangle / 2$ over wave space yields

$$
\begin{aligned}
\frac{3 \mathcal{K}^{(\mathrm{ver})}(t)}{\mathcal{K}(0)}= & {\left[\frac{(\chi+1)}{2}+\frac{3(\chi+1)}{2} \beta_{2}^{(e)}+\frac{3}{2} \beta_{0}^{(z)}-\frac{3}{7} \beta_{2}^{(z)}+\frac{1}{14} \beta_{4}^{(z)}-\frac{3}{32}(\chi-1)\left(3 W_{1}-W_{3}\right)\right] } \\
& +\left[\frac{15(\chi-1)}{4} \sum_{n=1}^{N_{0}} \sum_{\ell=2}^{n+2} \sum_{m=0}^{\ell-1}\left(\beta_{2 n}^{(e)} \frac{\pi}{2^{2 \ell-2}} a_{\ell-2, n}\right) \frac{(-1)^{(\ell+m-1)}(2 \ell-1) !}{m !(2 \ell-1-m) !} W_{2 \ell-2 m-1}\right] \\
& +\left[\frac{15}{16} \sum_{n=0}^{N_{0}} \sum_{\ell=3}^{n+3} \sum_{m=0}^{\ell-1}\left(\beta_{2 n}^{(z)} \frac{\pi}{2^{2 \ell-2}} a_{\ell-3, n}\right) \frac{(-1)^{(\ell+m-1)}(2 \ell-1) !}{m !(2 \ell-1-m) !} W_{2 \ell-2 m-1}\right] .
\end{aligned}
$$

Using the functional relation ${ }^{27}$

$$
W_{2 n-1}(2 N t)+W_{2 n+1}(2 N t)=\frac{2 n}{N t} W_{2 n}(2 N t),
$$

the expression of the horizontal contribution to kinetic energy 


$$
\mathcal{K}^{(\text {hor })}(t)=\frac{1}{2}\left(1+\frac{\chi}{2}\right) \mathcal{K}(0)-\frac{1}{2} \mathcal{K}^{(\text {ver })}(t)-\frac{1}{2} \mathcal{K}^{(\text {pot })}(t),
$$

is found as

$$
\begin{aligned}
\frac{3 \mathcal{K}^{(\mathrm{hor})}(t)}{\mathcal{K}(0)}= & \frac{7+\chi}{8}-\frac{3(1+\chi)}{4} \beta_{2}^{(e)}-\frac{27}{16} \beta_{0}^{(z)}+\frac{33}{56} \beta_{2}^{(z)}-\frac{3}{84} \beta_{4}^{(z)}-\frac{3(\chi-1)}{32(N t)} \pi W_{2}(2 N t) \\
& -\left[\frac{15(\chi-1)}{2} \sum_{n=1}^{N_{0}} \sum_{\ell=1}^{n+1} \sum_{m=0}^{\ell-1}\left(\beta_{2 n}^{(e)} \frac{\pi}{2^{2 \ell-2}} a_{\ell-1, n}\right) \frac{(-1)^{(\ell+m-1)}(2 \ell-1) !(\ell-m)}{m !(2 \ell-1-m) !} \frac{(\ell-1}{N t} W_{2 \ell-2 m}\right] \\
& -\left[\frac{15}{8} \sum_{n=0}^{N_{0}} \sum_{\ell=2}^{n+2} \sum_{m=0}^{\ell-1}\left(\beta_{2 n}^{(z)} \frac{\pi}{2^{2 \ell-2}} a_{\ell-2, n}\right) \frac{(-1)^{(\ell+m-1)}(2 \ell-1) !(\ell-m)}{m !(2 \ell-1-m) !} \frac{(\ell t}{N t} W_{2 \ell-2 m}\right]
\end{aligned}
$$

When $N t \ll 1$ both $\mathcal{K}^{(\mathrm{ver})}(t)$ and $\mathcal{K}^{(\text {hor })}(t)$ behave like $(N t)^{2}$. At large time, the oscillations for the vertical component decay with time proportionally to $t^{-1 / 2}$, whereas those of the horizontal component decay proportionally to $t^{-3 / 2}$. The long-time limit of the ratio $\mathcal{K}^{(\text {hor })} / \mathcal{K}^{(\text {ver })}$ depends on $\chi, \beta_{2}^{(e)}, \beta_{0}^{(z)}, \beta_{2}^{(z)}$, and $\beta_{4}^{(z)}$. Also, the long-time limit of $\mathcal{K}^{(\text {pol })} / \mathcal{K}^{(\text {ver })}$ depends on these parameters,

$$
\left(\frac{\mathcal{K}^{(\mathrm{pot})}}{\mathcal{K}^{(\mathrm{ver})}}\right)_{\infty}=\frac{3}{2}\left(1-\frac{[3(\chi+1) / 2] \beta_{2}^{(e)}+(1 / 4) \beta_{0}^{(z)}+(1 / 14) \beta_{2}^{(z)}+(1 / 14) \beta_{4}^{(z)}}{[(\chi+1) / 2]+[3(\chi+1) / 2] \beta_{2}^{(e)}+(3 / 2) \beta_{0}^{(z)}-(3 / 7) \beta_{2}^{(z)}+(1 / 14) \beta_{4}^{(z)}}\right) .
$$

Only for initial isotropic data [i.e., $\beta_{2 n}^{(e)}=\beta_{2 n}^{(z)}=0$ ], the above ratio reduces to $3 / 2$ independently of the value of the parameter $\chi$ (see Ref. 14). The development of $\mathcal{K}^{(\text {ver })}$ and $\mathcal{K}^{\text {(hor) }}$ normalized by $\mathcal{K}^{(\mathrm{ver})}(0)$ is shown on Fig. 1(b) for isotropic initial data with $\chi=0$.

Figures showing the development of energy components for anisotropic initial data are given in Sec. VI.

\section{Additional viscous effects}

It is also easy to account for the laminar viscous effects, provided that $P_{r}=\nu / \kappa=1$, i.e., the molecular Prandtl or Schmidt number. The solution of linearized equations which include viscosity can be obtained simply by multiplying the solutions (8) by $\exp \left(-\nu k^{2} t\right)$.

It follows that the evolution of energy components for the viscous case is expressed in terms of

$$
\begin{aligned}
& \alpha_{(\mathrm{vis})}(t)=\frac{1}{\mathcal{K}(0)} \int_{0}^{\infty} E(k) \exp \left(-2 \nu k^{2} t\right) d k, \\
& \beta_{(\mathrm{vis}) 2 n}^{(e)}(t)=-\frac{1}{15 \mathcal{K}(0)} \int_{0}^{\infty} E(k) e_{2 n} \exp \left(-2 \nu k^{2} t\right) d k, \\
& \beta_{(\mathrm{vis}) 2 n}^{(z)}(t)=\frac{4}{15 \mathcal{K}(0)} \int_{0}^{\infty} E(k) z_{2 n} \exp \left(-2 \nu k^{2} t\right) d k .
\end{aligned}
$$

The inviscid limit is recovered with $\alpha_{(\mathrm{vis})}=1, \beta_{(\mathrm{vis}) 2 n}^{(e)}$ $=\beta_{2 n}^{(e)}$ and $\beta_{(\mathrm{vis}) 2 n}^{(z)}=\beta_{2 n}^{(z)}$. Consequently, the long-time limit of the ratio $\mathcal{K}^{(\mathrm{pol})}(t) / \mathcal{K}^{\text {(tor) }}(t)$ is one even if the viscous terms are taken into account, and the long time limit of $\mathcal{K}^{\text {(hor) }}(t) / \mathcal{K}^{(\text {ver })}(t)$ and $\mathcal{K}^{(\text {pot })}(t) / \mathcal{K}^{\text {(ver) }}(t)$ has the same form as in an inviscid fluid, provided $\alpha=1, \beta_{2}^{(e)}, \beta_{0}^{(z)}, \beta_{2}^{(z)}$ and $\beta_{4}^{(z)}$ are replaced by $\alpha_{(\mathrm{vis})}, \beta_{(\mathrm{vis}) 2}^{(e)}, \beta_{(\mathrm{vis}) 0}^{(z)}, \beta_{(\mathrm{vis}) 2}^{(z)}$ and $\beta_{(\mathrm{vis}) 4}^{(z)}$, respectively. It follows that, for initial isotropic data, the long-time limit of the ratio in Eq. (47) is $3 / 2$.

\section{PHYSICAL INTERPRETATION OF THE PARAMETERS FOR INITIAL DATA: TYPICAL RESULTS}

Linear solutions call into play the sets of $\beta_{2 n}^{(e)}$ and $\beta_{2 m}^{(z)}$ parameters, which directly reflect the directional anisotropy and the polarization anisotropy, respectively. Is it possible to link these parameters to statistical quantities, such as those used in structure-based modelling? The answer is positive, at least at the lowest degree. Consequently, the choice of the most relevant parameters can be made on a more physical basis, and will be used for the last figures of this article.

\section{A. Dimensionless and tracefree second-order tensors}

The most classical anisotropy indicator is the deviatoric part of the Reynolds stress tensor, denoted as $b_{i j}$. Any similar tensor, dimensionless and tracefree, can be written

$$
b_{i j}=\frac{b_{(\mathrm{ver})}}{2}\left(3 n_{i} n_{j}-\delta_{i j}\right)
$$

in axisymmetric turbulence, characterized by a single (axial) component denoted $b_{(\text {ver })}=b_{i j} n_{i} n_{j}$ (see, e.g., Ref. 5). In accordance with the general equation (10), $b_{i j}$ can be split into a directional $b_{i j}^{(e)}$ component and a polarization one $b_{i j}^{(z)}$ (see Ref. 9), and this splitting allows a distinction between "dimensionality" and "componentality," as also proposed by Ref. 10.

Because of axial symmetry, only a single component per tensor, $b_{(\mathrm{ver})}^{(e)}$ and $b_{(\mathrm{ver})}^{(z)}$, will be needed. In addition, the definitions of spherically averaged spectra 


$$
\mathcal{K}(t) b_{i j}^{(e)}(t)=\int_{0}^{\infty} E(k, t) H_{i j}^{(e)}(k, t) d k
$$

and

$$
\mathcal{K}(t) b_{i j}^{(z)}(t)=\int_{0}^{\infty} E(k, t) H_{i j}^{(z)}(k, t) d k
$$

are also useful. $H_{i j}^{(e)}(k, t)$ and $H_{i j}^{(z)}(k, t)$ are derived from the spherically averaging Eq. (10), so that

$$
\int_{S_{k}} \hat{R}_{i j}(\boldsymbol{k}, t) d^{2} \boldsymbol{k}=2 E(k)((1 / 3) \delta_{i j}+\underbrace{H_{i j}^{(e)}(k, t)+H_{i j}^{(z)}(k, t)}_{H_{i j}(k, t)})
$$

is the $k$ spectral counterpart of the splitting of the Reynolds stress tensor $b_{i j}=b_{i j}^{(e)}+b_{i j}^{(z)}$. Once again, axisymmetry implies that only $H_{(\mathrm{ver})}^{(e)}$ and $H_{(v e r)}^{(z)}$ are needed.

The truncated expansion for both directional and polarization anisotropy at the lowest order for constructing the anisotropic part of $\hat{R}_{i j}(\boldsymbol{k})$ in (10) was recently found ${ }^{20}$ to be rigorously equivalent to the following tensorial expansion in terms of $\boldsymbol{k}, H_{i j}^{(e)}$, and $H_{i j}^{(z)}$,

$$
\mathcal{E}=-\frac{15}{2} \frac{E}{4 \pi k^{2}} H_{i j}^{(e)} \frac{k_{i} k_{j}}{k^{2}}, \quad Z=\frac{5}{2} \frac{E}{4 \pi k^{2}} H_{i j}^{(z)} N_{i}(-\boldsymbol{k}) N_{j}(-\boldsymbol{k}) .
$$

For instance, the five independent components of the (tracefree symmetric) tensor $H_{i j}^{(e)}$ have for exact counterpart the five coefficients $e_{2}^{l}(k), l=-2,-1,0,1,2$ of the lowest order decomposition of $\mathcal{E}$ in terms of classical scalar spherical harmonics.

In axisymmetric turbulence, the corresponding decomposition at the lowest order involves only $e_{2}(k)$ and $z_{0}(k)$, and the linkage reduces to

$$
e_{2}(k)=-15 H_{(\mathrm{ver})}^{(e)}, \quad z_{0}(k)=\frac{15}{4} H_{(\mathrm{ver})}^{(\bar{z})},
$$

so that $\beta_{2}^{(e)}=b_{(\mathrm{ver})}^{(e)}$ and $\beta_{0}^{(z)}=b_{(\mathrm{ver})}^{(z)}$. The coefficients $\beta_{2}^{(e)}$ and $\beta_{0}^{(z)}$ can therefore directly be identified to statistical indicators relevant in single-point structure based modeling.

Unfortunately, it is not possible to interpret in a similar way the coefficients at the four degree, for instance involved in Eq. (47). A possible way would be to use the spherically averaged spectrum of the rapid pressure-strain rate tensor.

\section{B. Final choice of initial parameters: Brief discussion of previous models}

Axisymmetric initial data can be created by an axisymmetric convergent duct in grid-generated turbulence. This suggests choosing initial data similar to those precalculated by irrotational axisymmetric RDT started with isotropic initial data. In their structure-based modeling approach, Kassinos et al. ${ }^{10}$ advocated for a simple equivalence of "dimensionality" and "componentality" for any irrotational strain, which corresponds to $-2 b_{i j}^{(e)}=b_{i j}^{(e)}+b_{i j}^{(z)}$ equating the deviatoric part of the "dimensionality structure tensor" and the deviatoric part of the Reynolds stress tensor. Although we do not think that such a relationship is really general, it is valid at least for weak anisotropy obtained by rapid distortion theory from strictly isotropic initial data, the first order timedevelopment giving ${ }^{20}$

$$
\begin{aligned}
& H_{i j}^{(e)}=\frac{1}{15}\left(1-\frac{k}{E} \frac{d E}{d k}\right) S_{i j} \Delta t ; \quad H_{i j}^{(z)}=-\frac{2}{5} S_{i j} \Delta t ; \\
& b_{i j}^{(e)}=-(1 / 3) b_{i j}^{(z)}
\end{aligned}
$$

independently of the applied "distorting" mean velocity gradient matrix, whose symmetric part is $S_{i j}$. A similar result, but with different proportionality of $b_{i j}^{(e)}$ to $b_{i j}^{(z)}$ can be found in the limit of weak anisotropy but large nonlinearity (using the sophisticated LRA closure theory), at least for large $k$ (see Ref. 29), as discussed in Ref. 20.

Retaining the last relationship in (50), which is also consistent with Ref. 10, it is possible to derive the two crucial parameters $\beta_{2}^{(e)}$ and $\beta_{0}^{(z)}$ from the single initial $b_{(\mathrm{ver})}$ : a positive value of $b_{(\mathrm{ver})}$ corresponds to a "cigar" type of Reynolds stress tensor, with the axial component larger than the two transverse ones, such as the axisymmetry resulting from the effect of an axisymmetric divergent duct. A negative value corresponds to a "pancake" type of Reynolds stress tensor, with the axial component smaller than the two transverse ones, such as the axisymmetry resulting from the effect of a convergent duct. Considering reasonable values of contraction in a hypothetical convergent duct, one finally chose

$$
b_{\text {ver }}=-0.04, \quad \beta_{2}^{(e)}=b_{\text {ver }}^{(e)}=0.02, \quad \beta_{0}^{(z)}=b_{(\mathrm{ver})}^{(z)}=-0.06 .
$$

Models from Refs. 30 and 12 used a tuned parameter to prescribe a proportionality between directional anisotropy and polarization anisotropy. In the more general model, the unique tuning parameter $a(k, t)$ in Ref. 30 can be interpreted as an $H^{(e)}$-to- $H^{(z)}$ partition ratio, so that a constant value of this parameter amounts to replacing $\beta^{(e)}$ by $(2+3 a)\left[\beta_{2}^{(e)}\right.$ $\left.+\beta_{0}^{(z)}\right] / 2$, and $\beta_{0}^{(z)}$ by $-2 a\left[\beta_{2}^{(e)}+\beta_{0}^{(z)}\right] / 5$. Anyway, the constant value of $a$ is not consistent with Eqs. (50) if spectra are considered.

The constant $\gamma$ plays a similar role of a $b_{i j}^{(e)}$-to- $b_{i j}^{(z)}$ partition ratio, in Ref. 12, whose model is a simplified version of the former, so that it amounts to replacing $\beta_{0}^{(z)}$ by $[6 /(7$ $-2 \gamma)]\left[\beta_{2}^{(e)}+\beta_{0}^{(z)}\right]$ and $\beta_{2}^{(e)}$ by $[(1-2 \gamma) /(7-2 \gamma)]\left[\beta_{2}^{(e)}+\beta_{0}^{(z)}\right]$.

In addition, the value $\gamma=1 / 2$ prescribed by Ref. 12 yields to discard the directional anisotropy, giving $\beta^{(e)}$ $=b_{(\mathrm{ver})}^{(e)}=0$ here, and more generally $b_{i j}^{(e)}=0$. This choice is inconsistent with the analyses presented previously (it is only used to enforce realizability) and will be rejected as unphysical. For instance, a pure return-to-isotropy was obtained by Ref. 11 in the case of rapid rotation, using this initial condition, contrary to cases where initial anisotropy results from a physical process.

\section{Selection of figures with typical parameters}

The evolution of the single relevant component $b_{(\mathrm{ver})}$ $=b_{i j} n_{i} n_{j}$ of the Reynolds stress tensor is shown on Fig. 2, for two initial cases, $b_{\text {(ver) }}(0)=-0.04$ and $b_{(\mathrm{ver})}(0)=0.04$. The former corresponds to initial data given by Eq. (51), as in all 


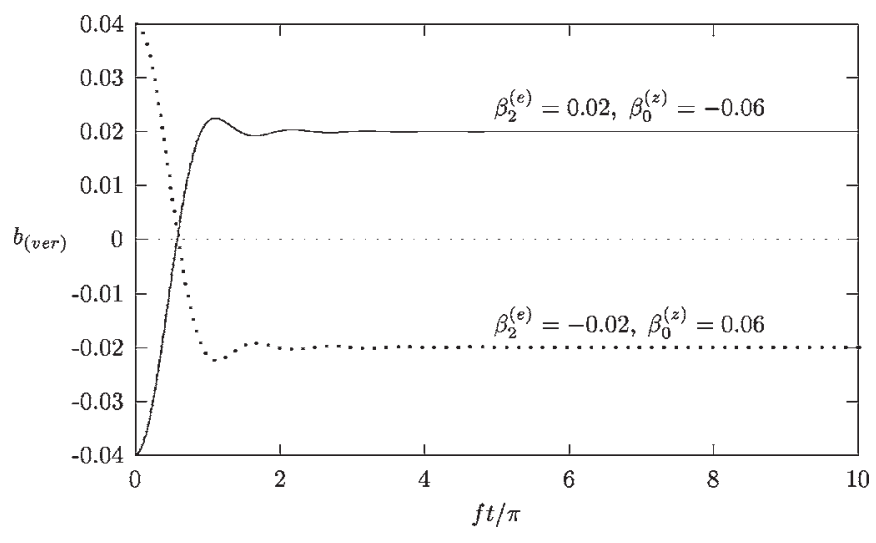

FIG. 2. Pure rotation, evolution of the axial (vertical) component of the deviatoric part of the Reynolds stress tensor.

of the following figures. The rapid change of sign of this indicator in about a quarter of a revolution $(f t / \pi \sim 1)$ is the most spectacular result of the linear solution. This behavior is completely missed in any Reynolds stress model, which gives a wrong zero value to the rapid pressure-strain term in this situation.

Corresponding evolution of typical energy components, divided by the initial vertical energy component, is collected in Fig. 3.

The same quantities are plotted on Fig. 4, but for the stratified case. The additional initial parameters $\beta_{2}^{(z)}$ and $\beta_{4}^{(z)}$, involved in the final ratios, as well as $\chi$, are taken with zero value for the sake of simplicity.

\section{CONCLUSION}

We have determined and analyzed, using the linear theory, the time evolution of the evolution of typical energy components, poloidal/toroidal, horizontal/vertical, and potential, for initial anisotropic axisymmetric data consistent with the dynamical equations in rapidly rotating and strongly stratified homogeneous flows. The initial anisotropic data have been generated using a modified spherical harmonic decomposition in terms of even Legendre polynomials and in agreement with mirror symmetry. ${ }^{18,19}$

For the stratified case, we have assumed that the initial spectral density of potential energy is proportional to that of kinetic energy $(\chi / 2$ is the proportionality coefficient), and the initial density fluxes are zero. We have shown that the poloidal part of the kinetic energy and the potential energy equilibrate after damped oscillations independently on the degree of the initial angular harmonic expansion even if viscous and diffusive terms with $P_{r}=1$ are taken into account. At large time, the oscillations for these energies, as well as those of the vertical part of kinetic energy, decay with time proportionally to $t^{-1 / 2}$, whereas those of the horizontal component decay proportionally to $t^{-3 / 2}$. The long-time limit of the ratio $\mathcal{K}^{\text {(hor) }} / \mathcal{K}^{\text {(ver) }}$ depends on $\chi$ and on the initial directional anisotropy and polarization anisotropy, more precisely on $\beta_{2}^{(e)}, \beta_{0}^{(z)}, \beta_{2}^{(z)}$, and $\beta_{4}^{(z)}$. In addition, the long-time limit of the ratio of the potential energy to the vertical kinetic energy depends on these parameters, and reduces to $3 / 2$ only for initially isotropic data.
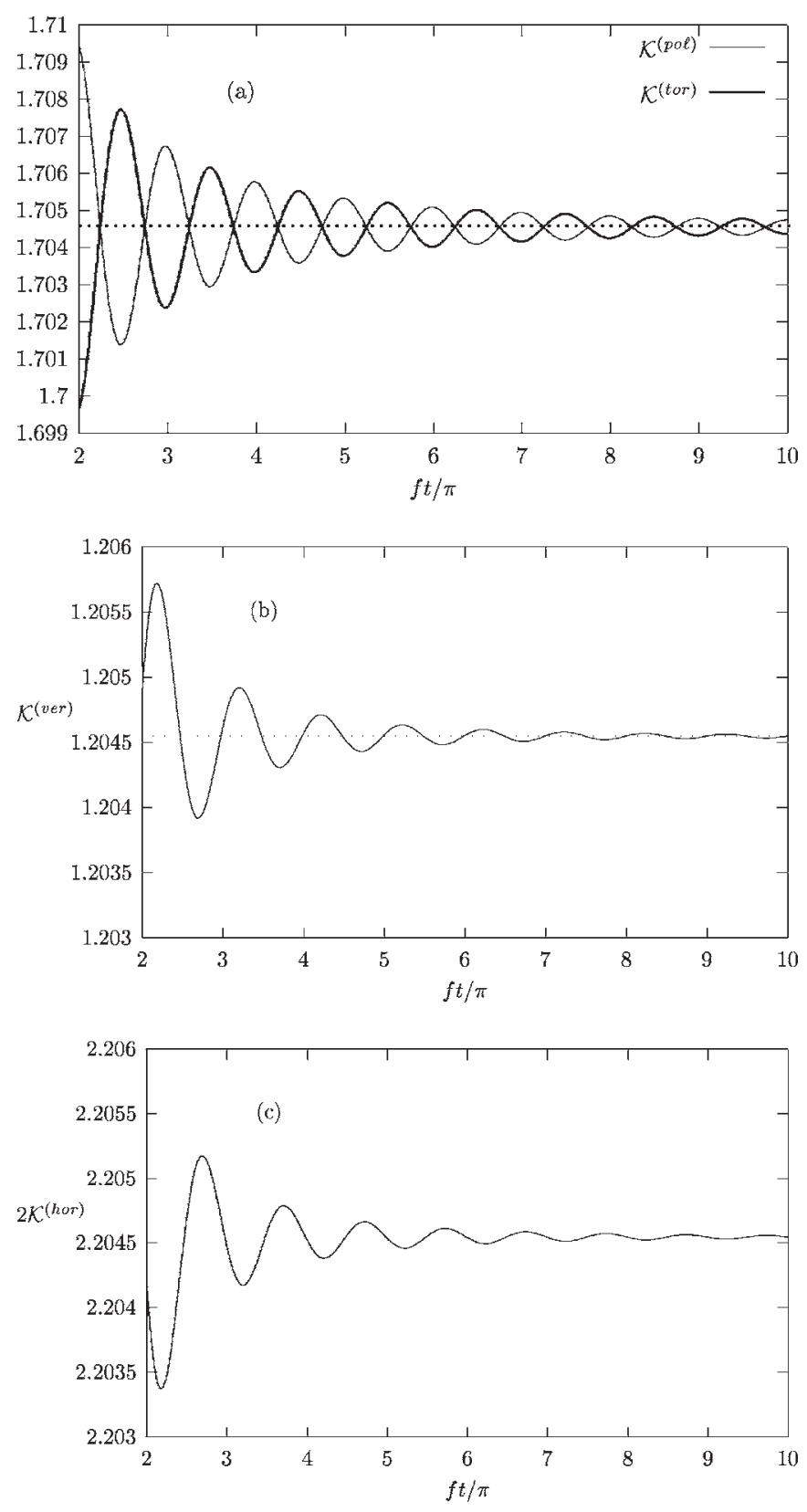

FIG. 3. Pure rotation, same anisotropic initial data, various energy components, Eqs. (24), (27), and (28).

For the rotating case, we have shown that the poloidal and toroidal contributions to kinetic energy equilibrate after damped oscillations independently on the degree of the initial angular harmonic expansion, and even in the presence of viscous terms. While, at large time, the envelope of oscillations for these parts of energy behave like $(f t)^{-2}$, those for the vertical and horizontal parts decrease like $(f t)^{-3}$, and the long-time limit of the ratio $\eta=\mathcal{K}^{\text {(hor) }} / \mathcal{K}^{\text {(ver) }}$ only depends on $\beta_{2}^{(e)}$, independently of the degree of the initial angular harmonic expansion. The evolution reflects a spectacular change of the sign of the single anisotropy component (here $b_{i j} n_{i} n_{j}$ ), which means a change of sign for the Lumley's invariant III.

Although similar results are found in both flow cases (stratified and rotating), the damping of rapid oscillations is significantly more rapid, or equivalently, the angular disper- 

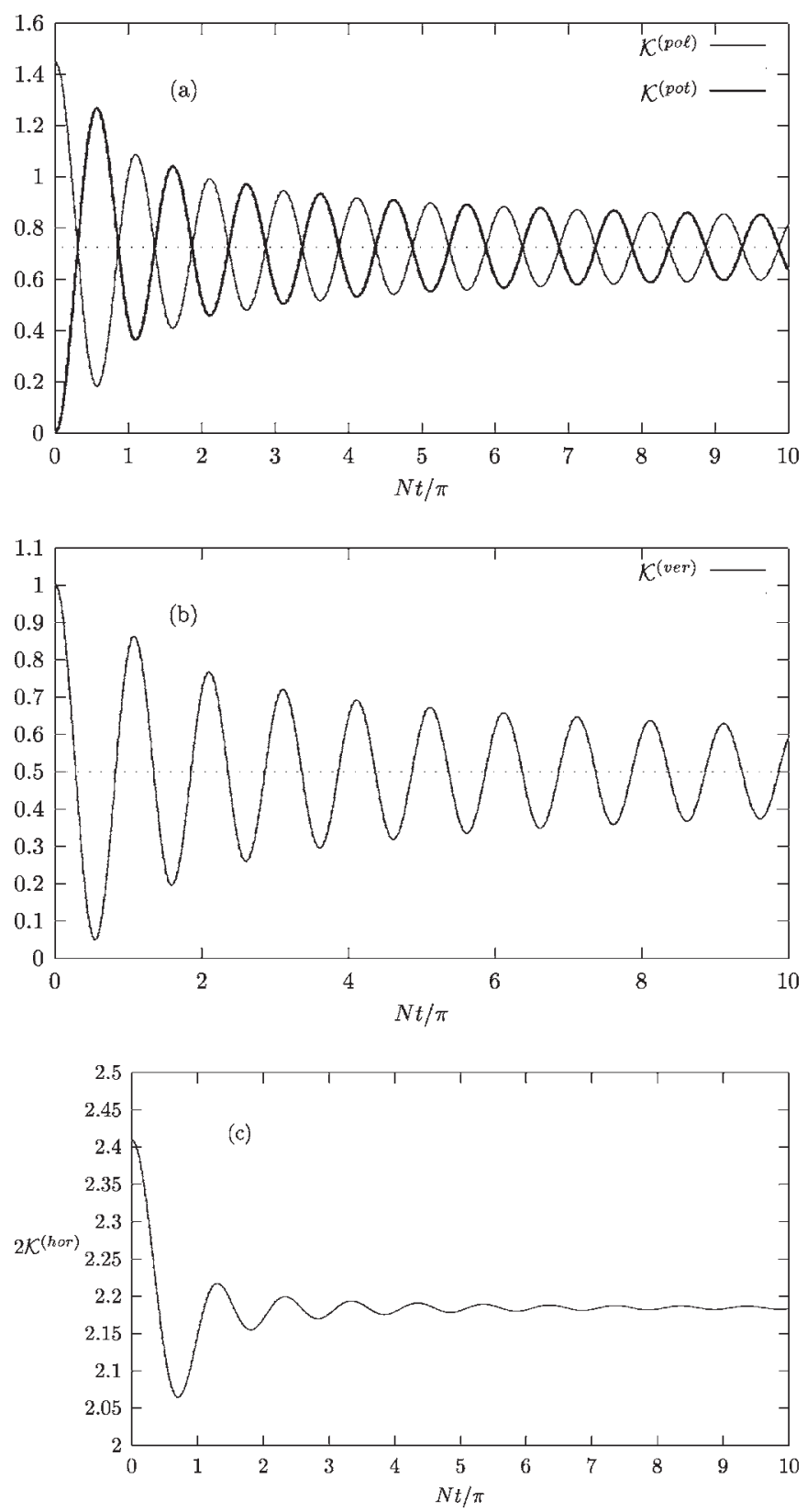

FIG. 4. Stable stratification, same anisotropic initial data, zero potential energy initially, Eqs. (40), (45), and (46).

sity is more pronounced, in the rotating case than in the stratified case.

The main parameters called into play for constructing initial data, which governs the long time evolution and asymptotic ratios, were finally expressed in terms of the relevant single (axial or vertical) component of directional $b_{i j}^{(e)}$ and polarization $b_{i j}^{(z)}$ components of the deviatoric part of the Reynolds stress tensor $b_{i j}$. Previous simplified models ${ }^{12,30}$ are shown to prescribe a $b_{i j}^{(e)}$-to- $b_{i j}^{(z)}$ ratio via a tuned constant. Only low order coefficients, such as $\beta_{2}^{(e)}$ and $\beta_{0}^{(z)}$ are concerned in the rotating case. Higher order parameters, such as $\beta_{2}^{(z)}$ and $\beta_{4}^{(z)}$, are involved in the stratified flow case. They could be related to the spectrum of the rapid pressure-strain rate tensor at $t=0^{+}$.

\section{APPENDIX: DETAIL ON LEGENDRE POLYNOMIALS AND WEBER FUNCTIONS \\ Legendre polynomials of even degree written in expanded form}

Because the polynomials $P_{0}(x)$ and $x P_{1}(x)$ and $P_{2}(x)$ can be written as

$P_{0}(x)=1, \quad x P_{1}(x)=1-\left(1-x^{2}\right), \quad P_{2}(x)=1-\frac{3}{2}\left(1-x^{2}\right)$,

and due to the following recurrence formulas (see Ref. 27):

$$
(n+1) P_{n+1}(x)-(2 n+1) x P_{n}(x)+n P_{n-1}(x)=0,
$$

we deduce that the Legendre polynomials of even degree can be written in expanded form as

$$
P_{2 n}=\sum_{\ell=0}^{n} a_{\ell, n}\left(1-x^{2}\right)^{\ell} \quad \text { with } a_{0, n}=1 .
$$

\section{Expression of the integral $I_{n-1}(2 N t)$ in terms of the Weber functions}

In this Appendix, we will establish the relation (39). We write the integral $I_{n-1}(2 N t)$ as

$$
\begin{aligned}
I_{n-1}(2 N t) & =\int_{-1}^{+1}\left(1-x^{2}\right)^{n-1} \cos \left(2 N t \sqrt{1-x^{2}}\right) d x \\
& =\int_{0}^{\pi} \sin ^{2 n-1} \theta \cos (2 N t \sin \theta) d \theta,
\end{aligned}
$$

or equivalently, using the representation of $\sin ^{2 n-1} \theta$ in terms of functions of multiples of the angle $\theta$ (see Ref. 27)

$$
\begin{aligned}
(-1)^{n+\ell-1} & \frac{(2 n-1) !}{\ell !(2 n-1-\ell) !} \sin (2 n-2 \ell-1) \theta \\
I_{n-1}(2 N t)= & \frac{1}{2^{2 n-2}} \sum_{\ell=0}^{n-1}(-1)^{n+\ell-1} \frac{(2 n-1) !}{\ell !(2 n-1-\ell) !} \int_{0}^{\pi} \sin (2 n \\
& -2 \ell-1) \theta \cos (2 N t \sin \theta) d \theta .
\end{aligned}
$$

The latter integral can be expressed in terms of the Weber functions that are associated with the Bessel functions. ${ }^{27,28}$ Indeed, the integral representation of the Weber functions $W_{2 n-1}(z)$ reads

$$
\begin{aligned}
W_{2 n-1}(z) & =\frac{1}{\pi} \int_{0}^{\pi} \sin [(2 n-1) \theta-z \sin \theta] d \theta \\
& =\frac{1}{\pi} \int_{0}^{\pi} \sin [(2 n-1) \theta] \cos (z \sin \theta) d \theta
\end{aligned}
$$

since

$$
\int_{0}^{\pi} \cos ((2 n-1) \theta) \sin (z \sin \theta) d \theta=0,
$$

so that 
$I_{n-1}(2 N t)=\frac{\pi}{2^{2 n-2}} \sum_{\ell=0}^{n-1}(-1)^{n+\ell-1} \frac{(2 n-1) !}{\ell !(2 n-1-\ell) !} W_{2 n-1-\ell}(2 N t)$

${ }^{1}$ C. Staquet and J. Sommeria, "Internal gravity waves: From instabilities to turbulence," Annu. Rev. Fluid Mech. 34, 559 (2002).

${ }^{2}$ Y. Kaneda, "Single-particle diffusion in strongly stratified and/or rapidly rotating turbulence," J. Phys. Soc. Jpn. 69, 3847 (2000).

${ }^{3} \mathrm{C}$. Cambon, "Turbulence and vortex structures in rotating and stratified flows," Eur. J. Mech. B/Fluids 20, 489 (2001).

${ }^{4}$ Y. Kaneda and T. Ishida, "Suppression of vertical diffusion in strongly stratified turbulence," J. Fluid Mech. 402, 311 (2000).

${ }^{5} \mathrm{C}$. Cambon and L. Jacquin, "Spectral approach to nonisotropic turbulence subjected to rotation," J. Fluid Mech. 202, 295 (1989).

${ }^{6}$ F. Bellet, F. S. Godeferd, J. F. Scott, and C. Cambon, "Wave turbulence in rapidly rotating flows," J. Fluid Mech. 552, 83 (2006).

${ }^{7}$ F. S. Godeferd and C. Cambon, "Detailed investigation of energy transfers in homogeneous stratified turbulence," Phys. Fluids 6, 2084 (1994).

${ }^{8}$ Y. Morinishi, K. Nakabayashi, and S. K. Ren, "Dynamics of anisotropy on decaying homogeneous turbulence subjected to system rotation," Phys. Fluids 13, 2912 (2001).

${ }^{9}$ C. Cambon, L. Jacquin, and J.-L. Lubrano, "Towards a new Reynolds stress model,” Phys. Fluids A 4, 812 (1992).

${ }^{10}$ S. C. Kassinos, W. C. Reynolds, and M. M. Rogers, "One-point turbulence structure tensors," J. Fluid Mech. 428, 213 (2000).

${ }^{11}$ N. N. Mansour, T. H. Shih, and W. C. Reynolds, "The effects of rotation on initially anisotropic homogeneous flows" Phys. Fluids A 3, 2421 (1991).

${ }^{12}$ T. H. Shih, W. C. Reynolds, and N. N. Mansour, "A spectrum model for weakly anisotropic turbulence,” Phys. Fluids A 2, 1500 (1990).

${ }^{13}$ J. C. R. Hunt, D. D. Stretch, and R. E. Britter, "Length scales in stably stratified turbulent flows and their use in turbulence models," in Stably Stratified Flow and Dense Gas Dispersion, edited by J. S. Puttock (Clarendon, Oxford, 1988), p. 281.

${ }^{14}$ H. Hanazaki and J. C. R. Hunt, "Linear processes in unsteady stably stratified turbulence," J. Fluid Mech. 318, 303 (1996).

${ }^{15} \mathrm{~L}$. van Haren, C. Staquet, and C. Cambon, "Decaying stratified turbulence: Comparison between a two-point closure EDQNM model and direct numerical simulations," Dyn. Atmos. Oceans 23, 217 (1996).
${ }^{16}$ J. R. Herring, "Approach of axisymmetric turbulence to isotropy," Phys. Fluids 17, 859 (1974).

${ }^{17}$ F. Waleffe, "Inertial transfers in the helical decomposition," Phys. Fluids A 5, 677 (1993).

${ }^{18}$ K. R. Sreenivasan and R. Narasimha, "Rapid distortion theory of axisymmetric turbulence," J. Fluid Mech. 84, 497 (1978).

${ }^{19} \mathrm{C}$. Cambon and C. Teissèdre, "Application des harmoniques sphériques à la représentation et au calcul des grandeurs cinématiques en turbulence homogène anisotrope," C. R. Acad. Sci., Ser. II: Mec., Phys., Chim., Sci. Terre Univers 301, 65 (1985).

${ }^{20}$ C. Cambon and R. Rubinstein, "Anisotropic developments for homogeneous shear flows," Phys. Fluids 18, 085106 (2006).

${ }^{21}$ S. H. Derbyshire and J. C. R. Hunt, "Structure of turbulence in stably stratified atmospheric boundary layers: Comparison of large eddy simulations and theoretical results," in Waves and Turbulence in Stably Stratified Flows, edited by S. D. Mobbs and J. C. King (Clarendon, Oxford, 1993), p. 23.

${ }^{22}$ D. J. Benney and A. C. Newell, "Random wave closure," Stud. Appl. Math. 48, 29 (1969).

${ }^{23}$ L. M. Smith and F. Waleffe, "Generation of slow large scales in forced rotating stratified turbulence," J. Fluid Mech. 451, 145 (2002).

${ }^{24} \mathrm{~J}$. J. Riley, R. W. Metcalfe, and M. A. Weissman, "Direct numerical simulations of homogeneous turbulence in density-stratified fluids," in Proceedings of the AIP Conference on Nonlinear Properties of Internal Waves, edited by B. J. West (American Institute of Physics, New York, 1981), p. 79.

${ }^{25}$ I. Arad, V. S. Lvov, and I. Proccaccia, "Correlation functions in isotropic and anisotropic turbulence: The role of the symmetry group," Phys. Rev. E 59, 6753 (1999).

${ }^{26} \mathrm{H}$. Hanazaki, "Linear processes in stably and unstably stratified rotating turbulence," J. Fluid Mech. 318, 303 (2002).

${ }^{27}$ I. S. Gradshteyn and I. M. Ryzhik, Table of Integrals, Series, and Products (Academic, New York, 1965).

${ }^{28}$ A. Erdelyi, W. Magnus, F. Oberhettinger, and F. G. Tricomi, Higher Transcendental Functions (McGraw-Hill, New York, 1953), Vols. 1-3.

${ }^{29}$ T. Ishihara, K. Yoshida and Y. Kaneda, "Anisotropic velocity correlation spectrum of homogeneous turbulent shear flow in a Lagrangian renormalized approximation," Phys. Rev. Lett. 88, 154501-1-4 (2002).

${ }^{30}$ C. Cambon, D. Jeandel, and J. Mathieu, "Spectral modelling of homogeneous nonisotropic turbulence," J. Fluid Mech. 104, 247 (1981). 